\title{
Author Index for Volume 104
}

\section{Aalfs, CM 1356}

Abadie, C 1517

Abbotts III, R 653

Abdah-Bortnyak, R 1649

Abenhardt, W 1071

Abernethy, AP 390

Abnet, CC 1511

Abud, L 1854

Acerini, CL 746

Acheson, N 1836

Adlard, J 1356

Aglietta, M 1686

Ahlbom, A 228

Ahmad, I 664

Ahmedzai, SH 1551

Ahn, JS 559

Ahson, G 1836

Aigelsreiter, A 1641

Aird, KM 1575

Aittomäki, K 1356

Akino, T 819

Akiyama, K 819

Akslen, LA 921

Al Ashari, M 1362

Alain, T 290

Alanko, T 599

Albert, A 989

Aldrich, A 1575

Alessi, DR 1116

Alexandre, J 1670

Ali, AMG 564, 693

Alibhai, S 1377

Al-Khyatt, W 1393

Allen, M 1675

Allen, NE 1487, 1493

Allgood, PC 1680

Al-Mahmood, S 496

Alman, BA 1452

Alonso, C 1106

Alonso, R 1356

Alsiary, R 1602

Altieri, DC 629

Amagasa, T 850

Amant, F 863

Amatschek, S 469

Amiano, P 1493

Amir, E 1377

Amira-Bouhidel, F 1739

Amodio, E 433

Ancona, E 427

Anders, M 1426

Andersen, PK 520

Anderson, J 175

Anderson, KS 1896

Anderson, R 1836

Andersson, S 110

Andreeff, M 91

Andrews, D 1365

Andrikopoulos, P 1151

Angeloni, C 248

Antoine, M 1739

Antoniou, AC 1356
Aoki, M 714

Ardizzoni, A 1372, 1929

Arigami, T 714

Arkenau, H-T 392

Armenoult, LSC 971

Armesilla, AL 1564

Arner, P 441

Ashcroft, M 1151

Ashdown-Barr, L 915

Ashley, S 1675

Ashton, KM 790

Asou, H 91

Atula, T 1890

Au, JSK 208

$\mathrm{Au}$, TCC 1000

Auer, G 110

Ausems, MGEM 1356

Austin, J 915

Avis, M 255

Avruch, J 24

Azzariti, A 769

Baba, H 300

Baccarini, M 229, 1240

Bachleitner-Hofmann, T 543

Bae, K-s 605

Baert-Desurmont, S 1517

Baeten, CG 37

Baglietto, L 524, 693

Bagni, B 1079

Bahl, A 1587

Bailey, D 1836

Baird, R 43

Bajetta, E 1256

Baker, JB 488

Balkwill, A 1487

Balmer, SM 726

Bandiera, E 1418

Banfield, A 587

Bao, Y 441

Bapat, B 1906

Baracos, VE 1288

Barbachano, Y 43

Barbanti, G 613

Barbany-Bustinza, G 1356

Barber, B 1848

Bardeesy, N 24

Bargagli, G 613

Barod, R 1151

Barricarte, A 1493

Barriuso, J 226

Barsky, SH 1628

Bassano, C 1372, 1929

Bast, A 437

Basu, B 1

Bates, TE 193

Batra, SK 1038

Bauernhofer, T 1641

Beard, R 620

Bearzi, I 1786

Beaudin, J 989

Becker, S 110
Beesley, J 1356

Bell, SM 1602

Bellanova, C 248

Bencko, V 1797

Benetou, V 1493

Bengtsson, N-O 899

Benítez, J 1356

Bennett, RL 571

Bennouna, J 413

Benoy, IH 1472

Beral, V 4, 1487

Berditchevski, F 1611

Bergamelli, S 1418

Bergmann, M 543

Berkhof, J 685

Bernardini, S 1770

Bernatsky, S 1478

Bernhard, H 948

Bernoud, R 1739

Berntsson, SG 1747

Bertheau, P 1739

Berthier-Vergnes, O 155

Bertolini, F 1079

Bethe, U 1691

Betts, G 1168, 1319

Bex, A 741

Beyene, J 1377

Bhandari, S 1236

Bhattacharyya, T 1151

Bieche, I 272

Bienkowski, M 968

Biernacka, KM 1587

Bignotti, E 1418

Bijnsdorp, IV 1185

Billan, S 1649

Billiot, F 1144

Bing, C 441

Biondo, A 43

Birch-Machin, M 1319

Birgisson, H 1619

Biscotti, T 1786

Bjørge, T 921

Blair, C 532

Blakely, J 68

Blann, A 1505

Blann, AD 1822

Blay, J-Y 1544

Blighe, K 1342

Blows, FM 693

Bocci, G 769, 1262

Böckelman, C 1890

Bocquet, C 1739

Boddy, AV 593, 1869

Bodin, I 110

Boeing, H 1493

Boffetta, P 1493, 1797

Boivin, M 989

Bonanni, B 1356

Bond, J 1602

Bonfiglio, TA 353

Bongaerts, M 496

Bonnier, P 332
Bono, P 599, 1686

Booth, CJ 1401

Borden, EC 957

Borgen, E 1434

Borriello, M 1716

Borthwick, K 324

Borup, R 830

Bose, D 1270

Boselli, S 1686

Bosma, AJ 1913

Bougeard, M 1517

Boult, JKR 83

Bourstyn, E 1739

Boutron-Ruault, M-C 1493

Bouville, A 181

Bowen, RL 120

Boye, K 1434

Boykin, C 781

Boyle, RW 1106

Bozic, B 1246

Bozzetti, C 1372, 1929

Bracarda, S 1256

Brandone, J-M 332

Brandt, R 635

Bray, F 178

Breene, RAL 746

Brennan, MF 1840

Brennan, P 1797

Brenner, AV 181

Brenner, H 1779

Brenton, JD 361

Brewer, C 1356

Brewster, DH 60, 1791

Bright, JJ 448

Brinkman, M 524

Broderick, P 1049

Broeders, MJM 910

Brotherton, JML 886

Brouste, V 1544

Brown, H 1319

Brown, J 1342

Brown, JM 1805

Brown, R 1313

Brown, S 1564

Browne, PV 281

Browne, S 1697

Bruchim, R 1049

Brudler, O 1071

Brugger, W 1071

Brugnara, S 1816

Brummendorf, TH 1691

Brunet, J 1356

Brüning-Richardson, A 1602

Bryant, HE 1098

Buchanan, DD 1906

Büchner, FL 1493

Buckland, G 1493

Bueno-de-Mesquita, HB 1493

Buffa, FM 1168

Bui, B 1544

Bukholm, I 1434

Burdach, S 948 
Burgers, JA 1325

Burke, W 750

Burns, P 1602

Burton, H 1656

Busch, DH 948

Butini, S 281

Buxton, ILO 1628

Byers, RJ 514

Byrne, C 587

Byrnes, GB 903

Bytheway, I 635

Bzhalava, D 178

Cadman, L 915

Cadron, I 863

Cahen-Doidy, L 1739

Cai, C 1319

Caiazza, F 338

Cailleux, A-F 1517

Cairns, BJ 4

Cairns, DA 1602

Caldas, C 693

Caldon, LJM 1551

Califano, R 750

Caligo, MA 1356

Calvert, H 593

Calza, S 1418

Cameron, D 1529

Camilloni, L 248

Camisa, R 1372, 1929

Campbell, FC 1007

Campbell, H 1529

Campbell, NC 1697

Campiani, G 281

Camps, C 1168

Canovas, D 1098

Canu, B 1262

Capellá, G 735, 1519

Capelle, L 1854

Capelli, S 1372

Capparucci, P 248

Capriotti, F 1185

Caradec, J 1056

Card, T 193

Carmichael, J 750

Carozzi, F 248

Carpenter, LM 1227

Carpenter, R 120

Carstensen, J 899

Carter, R 353

Cartwright, JE 83

Carvajal-Carmona, L 369

Casali, PG 1686

Cascinu, S 1786

Cassidy, J 1564

Cavalli, S 1929

Cesario, A 1877

Chakraborty, S 448, 1038

Challen, C 1319

Chan, ATC 1000

Chan, CML 1000

Chan, KW 841

Chan, S 407

Chan, YP 841

Chang, H-M 605

Chang, MS 166

Charles, KA 1288

Chau, I 43
Chau-in, S 1313

Chen, EX 756

Chen, X-C 1356

Chen, Y 308, 892, 1013

Chen, Y-H 781

Chenevix-Trench, G 1356

Cheng, CWS 941

Cheng, J 1401

Cheng, KK 369

Cheng, T 1410, 1931

Cheriyath, V 957

Cherny, SS 369

Chester, JD 75

Cheung, AHK 1000

Cheung, KL 1393

Cheung, KT 790

Cheung, MT 1000

Chhaya, V 43

Chia, KS 871

Chia, VM 763

Chiarappa, P 769

Chiarion-Sileni, V 1816

Chie, W-C 587

Chiras, J 1854

Chiriacò, G 613

Chiyomaru, T 376, 808

Cho, SJ 1126

Choi, IJ 1126

Choi, MS 559

Choi, S-c 369

Choi, SH 1027

Choi, SY 1730

Choi, Y 1730

Choi, YJ 166

Chong, TW 941

Chow, W-H 537, 1511, 1797

Chowdhury, S 407, 741, 1656

Christensen, K 520

Christensen, S 419

Christison, J 915

Chua, W 1288

Chuang, S-C 1178

Chuang, TH 1410, 1931

Chung, CC 369

Chung, IY 1730

Ciardiello, F 427

Clark, DI 60

Clark, EA 488

Clark, SE 120

Clark, SK 1236

Clarke, AE 1478

Clarke, SJ 1288

Clavel-Chapelon, F 1493

Coco, P 1686

Coghill, AE 763

Coit, DG 1840

Colditz, GA 882

Coleman, RE 1665

Colin, S 496

Collins, KA 1551

Collins, SI 1500

Colucci, G 1079, 1816

Comamala, M 989

Conca, R 613

Confortini, M 248

Congedo, MT 1877

Conlon, S 480

Connor, T 1151
Conrad, H 948

Constantinidou, A 1675

Conte, PF 1079

Conway, EL 886

Cook, M 1356

Cooke, SL 361

Coombes, RC 1342

Cooper, PA 75

Corso, G 1770

Costalat, R 1854

Cottet, V 1493

Cotton, S 255

Couch, FJ 1356

Courtney, R 1862

Couto, E 1493

Crafa, P 1372, 1929

Crawford, RAF 361

Cremolini, C 1262

Crespi, CM 228

Cretella, M 1770

Cronin-Fenton, DP 188, 1804

Crowe, F 1493

Cruickshank, M 255

Cubie, HA 1221

Cui, T 1013

Cullis, ER 83

Cummings, J 719

Cunningham, D 43

Curran, WJ 1365

Currie, MJ 51

Currow, DC 390

Curzon, B 1356

Cuschieri, K 1221

Cvitkovic, E 272

Dabelsteen, S 830

Dachs, GU 51

D'Addetta, A 248

Daduang, J 1313

Daemen, A 863

Daftary, D 1906

Dahiya, R 308, 892

Dahm, CC 1493

Dai, W 1313

Dal Maso, L 1207

D'Alessio, G 1716

Dallas, NA 1270

Dalton, SO 188, 1804

Damery, S 927

Danesi, R 1262

Darbyshire, J 1529

Darcy, KM 353

Darling, JL 1564

Darmanis, S 1619

Das, N 1836

Davidson, R 1356

Davies, JM 1067

Davis, M 875

Dawson, S-J 693

Day, FL 265

de Bazelaire, C 1739

de Bono, JS 1

De Braud, F 1686

de Gelder, R 1214

de Jong, S 1278

de Koning, HJ 1214

de la Cour, ZD 1202

de la Fouchardière, A 155
De Lorenzo, C 1716

De Marco, G 1854

de Mol, BAJM 1325

De Moor, B 863

De Pas, T 1686

de Roquancourt, A 1739

De Rubertis, G 613

De Salvo, GL 1816

de Thé, H 1739

De Vita, F 427

de Vries, EG 1278

Dearnaley, D 175

Decarli, A 1207

Dekker, E 37

Dekker, LV 653

Del Bianco, P 1816

Del Bufalo, D 769

Del Genio, A 427

Del Giovane, C 1079

Del Tacca, M 769

Del Vecchio Blanco, G 1770

Delacotte, N 1056

Dell'Aniello, S 1558

den Heeten, GJ 910

Deng, G 892

Dent, T 1656

Depenni, R 1079

Dernede, U 407

Despierre, E 863

Devi, GR 1575

Devriese, LA 1913

Dewar, J 1246

Di Cecilia, S 1770

Di Desidero, T 1262

Di Iulio, J 265

Di Maio, M 427

Di Martino, N 427

Di Paolo, A 1262

Di Pierro, C 248

Diamond, T 1007

Dicker, AP 1365

Dicks, E 1906

Dickson, MA 1862

Dieras, V 1670

diGioia, D 1071

Dillner, J 178

Dimitropoulou, P 875

Dirix, LY 1472

Dite, GS 903

Dive, C 719

Doki, Y 707

Dolled-Filhart, M 68

Domchek, S 1384

Domchek, SM 1356

Donaghy, M 1221

Donders, R 910

Dong, Y 893

Donne, AJ 324

Donovan, JL 875

Douglas, F 1356

Downing, S 1356

Dowswell, G 927

Dowty, JG 903

Draisma, G 1214

Draper, G 228

Dredge, K 635

Drozdovitch, VV 181

D’Souza, G 1896 
Duanmu, J 1401

Duce, SL 1116

Duffy, SW 120, 871, 1656, 1680

Duhamel, A 1544

Duiker, EW 1278

Dutta, D 488

Dvrokind, I 1649

Dziunycz, P 1106

Easton, D 175

Easton, DF 1356, 1656

Eatock, M 593

Eccles, D 1356

Edwards, DR 664

Edwards, J 1920

Edwards, R 915

Eeles, RA 175, 578, 1356, 1656

Eger, A 469

Ehrencrona, H 1356

Eisen, A 1384

Eisenhauer, EA 756

El-Bahrawy, MA 1313

Elder, DJE 1755

Ellis, IO 693, 1393

Ellis, LM 1270

Elsir, T 1747

El-Tanani, M 1007

Endo, Y 798

Engeset, D 1493

English, DR 524

Enokida, H 376, 808

Erichsen, R 1202

Erlander, MG 1762

Escudier, B 1144

Espié, M 1739

Esteban, MA 1151

Evans, DG 578, 1356

Evans, P 281

Evans, T 1505

Everard, J 1665

Eveson, JW 1459

Evseenko, VV 181

Facchetti, F 1418

Faivre, S 272

Falchetti, M 1418

Falcone, A 1262, 1786

Fallowfield, L 1535

Faloppi, L 1786

Fan, F 1270

Fan, X-E 781

Farace, F 1144

Farella, A 427

Farewell, D 1535

Farewell, V 1535

Farkas, DK 1202

Farrugia, D 1067

Faulstich, F 1334

Fearon, KCH 441

Federici, G 1770

Feldman, D 1049

Feliu, J 226

Feliubadaló, L 1356

Ferguson, M 1877

Ferrari, P 1493

Ferraroni, M 1207

Ferro, V 635

Fiaschi, AI 613
Fields, RC 1840

Figueiredo, C 198

Fina, F 332, 1364

Fiocchi, F 1079

Fioravanti, A 769, 1262

Fiorentino, F 1085

Fischer, PM 653

Fisher, AL 460

Fitamant, J 24

Flandre, E 1739

Flatmark, K 1434

Fleming, J 664

Fleming, S 1116

Flint-Richter, P 1049

Fodstad, Ø 1434

Foley, K 390

Fombon, IS 1564

Fontana, A 1079

Ford, E 1535

Formosa, A 1770

Fornander, T 899, 1762

Forster, JA 1135

Forsyth, PA 290

Fort, E 1836

Fortuzzi, S 1356

Foulis, AK 726

Foulkes, WD 1384, 1478

Franceschi, S 1207

Francini, E 613

Francini, G 613

Frater, A 915

Frebourg, T 1517

Fredericksen, Z 1356

Freier, W 1071

Friedland, PL 1246

Friedrich, RE 138

Friend, E 587

Friis, S 188, 1804

Friis-Hansen, L 830

Frost, D 1356

Frost, K 1303

Frumento, P 1262

Frydenberg, M 934, 1930

Fujiwara, K 353

Fujiwara, Y 707

Fukuhara, H 1349

Fukushima, M 1185

Fung, SS 369

Furukawa, M 1160

Furuyama, N 798

Galizia, E 1786

Galizia, G 427

Gallinger, S 1906

Gambier, N 1238

Ganesan, R 1505, 1611

Gao, D 441

Gao, F 871

Gao, W 1704

Gao, Y-T 1511

Garant, KA 290

Garantziotis, S 542

Garcia-Barceló, M-M 369

García-Martínez, JM 1116

Gardini, G 1372, 1929

Garlick, DS 629

Garnæs, E 830

Gasparro, D 1372, 1929
Gattens, M 746

Gatter, KC 1877

Gaur, P 1270

Gee, HE 1168

Geisler, J 1059

Gelderblom, H 37

Georgoulias, V 316

Gershman, S 1384

Gerster, M 520

Gerunda, GE 1079

Ghadirian, P 1384

Ghaneh, P 1440

Giacchetti, S 1739

Giampieri, R 1786

Gibson, W 1575

Gilbert, D 407

Gilbert, FJ 578

Gilbert, R 875

Giles, GG 524, 903

Gillatt, D 1587

Gillet, P 1238

Giorgi Rossi, P 248

Gitsch, G 1071

Glabbeke, MV 1544

Glimelius, B 1619

Godwin, A 1356

Goedert, JJ 433

Goel, HL 629

Goggins, M 1296

Gök, M 685

Golby, S 1836

Goldenring, JR 33

Goldwasser, F 1670

Gonda, TJ 635

Gönen, M 1840

Gonzalez, CA 1493

Gonzalez-Perez, RR 128

Goodman, KA 1840

Goodyer, M 281

Gordon, C 1478

Gordon, J 1836

Gordon, N 1611

Gore, ME 399, 750

Gornall, R 1611

Goto, Y 464

Grabar, S 1670

Grasl-Kraupp, B 1303

Gratus, C 927

Graubard, BI 433

Gravells, P 1098

Gray, NM 255, 1697

Grazzini, G 248

Green, AR 1393

Green, J 1487

Green, JA 1521

Green, RC 1906

Green, S 68

Greenberg, D 564, 1810

Greenfield, S 927

Gregersen, H 1202

Grelier, G 387

Greystoke, A 719, 750

Griesbacher, A 1641

Grieve, R 927

Griffin, M 593

Griffioen, AW 1185

Grignagni, G 1686

Grimm, C 223
Gromiec, J 1797

Groselj-Strele, A 1641

Gross-Goupil, M 1144

Grosse-Kracht, S 469

Gruber, SB 735, 1519

Grulich, A 886

Grusch, M 1303

Gualberto, A 68

Guichard, S 1116

Guida, M 1816

Guigay, J 1691

Guilfoyle, MR 1810

Guillén-Ponce, C 224

Guillevin, R 1854

Guilmain, W 496

Guinó, E 735

Gulliford, M 1704

Guo, S 128

Guttery, DS 1342

Guy, M 175

Gwak, G-Y 559

Habas, C 1854

Haddad, R 1896

Haenen, GRMM 437

Hagihara, J 228

Haglund, C 1890

Hagström, J 1890

Hague, A 1459

Haick, H 1649

Hakim, M 1649

Hall, AL 175

Hall, GD 1602

Hall, JS 971

Hall, SJ 1697

Hallmans, G 1493

Hamada, J-I 798

Hambleton, J 413

Hamdy, FC 875

Hamilton, P 1007

Hamilton, R 1529

Hamilton, W 934, 1930

Hammond, E 635

Hampson, IN 324

Hampson, L 324

Han, C 1822

Hancock, BW 1665

Handley, P 635

Handra-Luca, A 1296

Hank Juo, S-H 1178

Hansen, RP 934, 1249, 1930

Harao, M 300

Harbison, CT 488

Hareyama, M 1724

Hårklau, L 1434

Harnden, P 1135

Harris, AL 1168, 1822

Harris, F 1810

Harriss, D 175

Hartle, J 746

Hartmann, C 1747

Hartschuh, W 1334

Harvey, BJ 338

Hatae, M 353

Hatch, M 181

Hatschek, T 899

Haug, U 1779

Hauler, F 1106 
Hauptmann, M 1913

Hava, N 1342

Haward, R 1529

Hayashi, Y 798

Hayden, P 281

Hayes, DN 545

Haynes, K 1810

Häyry, V 1890

Hayward, N 1067

$\mathrm{He}, \mathrm{X} 235,324$

Healey, S 1356

Heer, R 1869

Heijnsdijk, EAM 1214

Heinemann, V 1071

Helgason, HH 1913

Hellman, K 110

Hellman, U 110

Hellström, A-C 110

Henrich, H 345

Heo, JS 1027

Hetzel, MR 1755

Hickson, ID 653

Hida, K 819

Hida, Y 819

Higashino, F 819

Higginson, IJ 1704

Hill, ADK 338

Hill, C 1144

Hinch, E 1055

Hinkal, GW 387

Hinoda, Y 308

Hinz, A 345

Hiraike, H 1349

Hirata, H 308

Hirata, S 300

Hirte, HW 756

Hixon, ML 68

Ho, JW 369

Ho, S 369

Hoang-Xuan, K 1854

Hocking, JS 886

Hodge, A 524

Hofbauer, GF 1106

Hogervorst, FB 1356

Hoh, L 1098

Hohenberger, P 1544

Holland, R 910

Hollenbeck, AR 537

Holly, JMP 1587

Holmlund, B 1762

Holt, S 1551

Homer, J 1168

Hong, H 1452

Hong, S-M 1296

Hong, YS 605

Hopper, JL 524, 903

Horgan, PG 726

Horikawa, T 1160

Horne, LS 460

Hosking, FJ 1049

Hospers, GAP 1193

Hotte, SJ 756

Hou, M-F 1178

Houlston, RS 369, 1049

Hruban, R 1296

Hsieh, C-C 629

Hsu, SM 982

$\mathrm{Hu}, \mathrm{Z} 1401$
Huang, PH 982

Huang, S 235

Huber, O 1013

Hughes, A 719

Hughes, G 1836

Hui, EP 1000

Hui, SM 369

Hui, TC 369

Huiart, L 1558

Hundsberger, H 469

Hundt, S 1779

Huntsman, D 693

Hurtz, HJ 1071

Hurwitz, H 413

Hussaini, HM 460

Hutson, R 1602

Hutson, T 741

Hutton, J 578

Huynh, H 941

Ikeda, Y 1349

Ikushima, H 241

Ikuta, Y 300

Illidge, TM 719

Ilson, DH 1840

Im, Y-H 559

Imai, K 300

Imamura, T 1882

Inaba, T 91

Inenaga, S 1882

Ingram, SM 1575

Innocenti, R 427

Inoue, M 300

Iossa, A 248

Irie, A 300

Irlam, JJ 971

Isaacs, C 1356

Ishigami, S 714

Ishizu, A 241

Ismail-Khan, R 1828

Isoniemi, H 599

Iurisci, I 1670

Izatt, L 1356

Izumi, H 1882

Izumi, K 505

Jacobs, H 437

Jacobsen, HJ 1434

Jacques, N 1144

Jager, A 1356

Jain, M 1038

Jakes, RW 871

Jalava, T 1686

Jang, G 605

Jang, K-T 1027

Janin, A 1739

Janout, V 1797

Jaskolski, DJ 968

Javot, L 1238

Jearanaikoon, P 1313

Jefferies, SJ 1810

Jeffery, C 1810

Jefford, M 265

Jenab, M 1493

Jenkins, MA 903

Jenkins, V 1535

Jensen, AB 419

Jerevall, P-L 1762
Jernström, H 1356

Ji, B-T 1511

Jimenez-Linan, M 361

Jin, L 91

Jirström, K 1619

Joensuu, H 1686

Johannessen, H-O 1434

Johannsson, OT 1356

Johansson, C 1619

Johansson, I 1493

Johnson, CD 587

Johnson, N 1836

Johnston, M 1697

Johnston, PG 480

Johnston, RN 290

Johnston, S 1675

Johnston, SJ 1393

Johnstone, AP 83

Jonat, W 1071

Jones, J 927

Jones, JL 120

Jones, L 407

Jones, R 741

Jubb, AM 1877

Judson, I 1544

Jung, J 166

Kaaks, R 1493

Kai, M 1724

Kaizaki, Y 1160

Kaklamanis, L 316

Kakuguchi, W 819

Kalaycio, ME 957

Kalimutho, M 1770

Kalland, KH 921

Kamangar, F 1511

Kamdar, RP 1724

Kamishima, T 241

Kanakasabai, S 448

Kanayama, H 241, 505

Kang, BH 629

Kang, E 1730

Kang, ES 559

Kang, Y-K 605

Kannappan, V 1564

Kaplan, R 1529

Kappeler, C 1686

Karami, S 1797

Karp, DD 68

Karpathakis, A 43

Karran, P 1869

Kataoka, K 593

Kato, S 1349

Kauderer, J 353

Kawahara, K 376

Kawakami, K 376, 808

Kay, EW 480

Kearins, O 1680

Keates-Porter, S 1836

Keating, PJ 790

Kefford, R 392

Keilholz, U 1691

Keller, E 664

Kelsen, DP 1840

Kelsey, KT 1896

Kennedy, H 1393

Kerber, A 1691

Kern, F 229, 1240
Kerr, D 1529

Keski-Säntti, H 1890

Kesty, NC 1762

Kettner, E 1071

Key, TJ 6

Khambata-Ford, S 488

Khan, G 1362

Khan, MN 1151

Khan, OA 750, 1822

Kharbili, MEI 155

Khatri, G 892

Khaw, K-T 1493

Kheifets, L 228

Kikkawa, N 376

Killian, A 1517

Kim, CG 1126

Kim, EJ 1730

Kim, H-S 605

Kim, HL 643

Kim, IA 1730

Kim, JH 1730

Kim, K-p 605

Kim, KH 559

Kim, M 290

Kim, NK 1126

Kim, S-W 1730

Kim, TW 605

Kim, WH 166

Kim, YH 166

Kim, YII 1027

Kim, YJ 1730

Kim, YW 1126

Kim-Sing, C 1384

Kimura, H 700

Kimura, S 91

Kinlen, L 12

Kiriakidis, S 1151

Kirichek, O 1067

Kita, Y 714

Kleibeuker, JH 37

Kline, K 101

Klomp, M 1020

Kloor, M 1334

Knight, JA 1906

Knijn, N 1020

Knoll, C 469

Knösel, T 1013

Knowles, MA 75, 1135

Knudsen, AB 1779

Ko, JMY 841

Ko, YS 166

Koffijberg, H 1325

Kohno, K 1882

Kollarova, H 1797

Kondo, C 856

Kondo, M 819

Kondo, S 1160

Kong, S-Y 1126

Konishi, N 700

Konopleva, M 91

Konstantopoulou, I 1356

Kooi, N 1278

Kornfeld, J-W 138

Koschel, A 1426

Kostourou, V 83

Koszik, F 469

Kote-Jarai, Z 175

Kotsori, A 1675 
Koyama, S 1349

Krautkrämer, E 1334

Krishnan, K 524

Kroll, ME 1227

Kruitwagen, RF 221

Kruse, AJ 221

Kruyt, FAE 1185

Kuan, R 1246

Kuhns, MA 957

Kullar, PJ 1810

Kumar, S 1038

Kuntz, KM 1779

Kurahara, H 714

Kuroda, J 91

Kurokawa, Y 707

Kuroshima, T 819

Kurosu, T 819

Kuten, A 1649

Kuwata, K 1594

Kwan-Lim, GE 578

La Vecchia, C 1207

LaBaer, J 1896

Labianca, R 1786

Laccetti, P 1716

Lacevic, M 1828

Lacey, M 1221

Lachuer, J 155

Lackner, A 1303

Lagiou, P 1493

Lagoudaki, E 316

Lagrasta, CA 1372, 1929

Lai, PBS 1000

Lajer, CB 830

Lalloo, F 1356

Lam, MYY 1000

Lamartine, J 155

Lamont, FR 75

Lane, JA 875

Langer, CJ 68

Langers, AM 37

Langner, C 1641

Languino, LR 629

Larkin, JMG 399, 407, 750

Lasa, A 1356

Lash, TL 188, 1804

Lattanzi, A 248

Lau, PY 369

Lau, WKO 941

Laughlin, A 756

Laughton, CA 653

Launay, O 1670

Laurent, D 1686

Law, M 886

Lawler, MP 281

Lawrence, G 1680

Lawrence, YR 1365

Le Chevalier, F 1670

Le Naour, F 155

Leach, MO 578

Lebon, P 1670

Lee, AJ 1697

Lee, BL 166

Lee, HE 1730

Lee, J-L 605

Lee, JE 559

Lee, JH 1126

Lee, JK 1027
Lee, JY 1027, 1126

Lee, KH 1027

Lee, KT 1027

Lee, PWK 290

Lee, SS 605

Lee, VHM 1000

Lee, Y-f 369

Leek, R 1168

Legrand, E 496

Legres, L 1739

Lehmann-Che, J 1739

Leighl, NB 413

Leonardi, F 1372, 1929

Leong, HS 971

Leong, T 265

Lester, N 1529

Leunen, K 863

Leung, HY 664

Levine, L 353

Lew, JQ 537

Lewandowski, P 1055

Li, D 68

Li, H 1762

Li, H-L 1511

Li, J 1523

Li, J-L 841

Li, L 19

Li, PJ 841

Li, Z 1882

Lian, JB 629

Liao, D 1410, 1931

Liao, S-Y 353

Liao, YH 982

Liberski, PP 968

Ligtenberg, MJ 1356

Liljegren, A 1356

Lim, GH 871

Lim, H-S 605

Lim, L 741

Limpaiboon, T 1313

Lin, J 464

Lin, S-R 1178

Lindman, H 899

Lindor, NM 1356

Lindström, MS 1747

Linecker, A 1641

Linton, K 719

Lippi, G 1234

Little, J 255

Liu, J 892, 1241

Liu, J-F 175

Liu, P 1564

Liu, R 369

Lloyd, A 1049

Lococo, F 1877

Lofts, F 407

Logie, C 554

Loken, SD 290

Loman, N 1356

Lomnytska, MI 110

Lonardi, S 1418

Long, GV 392

Longtine, J 1896

Löning, T 138

Lopes, C 198

Lophatananon, A 175

Lorch, J 1896

Loric, S 1056
Lorigan, P 750

LoRusso, PM 1862

Losekoot, N 1185

Lou, Z 19

Loulergue, P 1670

Loupakis, F 1262, 1786

Lowenthal, RM 228

Lu, C-Y 1178

Lu, J 1270

Lu, Q 781

Lubin, J 181

Lucas, R 469

Luckyanov, N 181

Ludeman, L 1611

Luk, LY 1000

Lund, E 1493

Lundin, J 1890

Lunet, N 198

Lung, ML 841

Luo, Y 1410, 1931

Luppi, G 1079

Lyerly, HK 1575

Lyman, R 1221

Lynch, HT 1384

Lyons, D 915

Ma, BBY 1000

Ma, X-J 1762

Ma, YT 1500

MacArthur, S 361

MacDonagh, B 281

Machesky, L 664

Macleod, U 1697

Maddala, T 488

Maders, F 719

Madhusudan, S 653

Madsen, M 520

Maginn, EN 281

Maishi, N 819

Maitland, N 673

Majid, S 892

Mäkinen, LK 1890

Makino, T 707

Malavasi, N 1079

Malmström, P-O 899

Maloney, S 1611

Mandolesi, A 1786

Manganelli, A 613

Mangia, A 769

Mania, E 248

Manjer, J 1493

Manoukian, S 1356

Mantle, M 741

Margison, GP 750

Markowitz, D 1410, 1931

Marom, O 1649

Marquez, R 664

Marreaud, S 1544

Marrelli, D 1770

Marsili, LM 248

Martens, JHA 554

Martin, FL 790

Martin, P-M 332, 1364

Martin, RM 875

Martinelli, E 427

Martin-Hirsch, PL 790

Masi, G 1262

Masur, K 345
Masuzawa, T 707

Masyakin, VB 181

Matera, A 265

Mates, D 1797

Mathoulin-Pelissier, S 1544

Mathus-Vliegen, EM 37

Matsuda, R 376

Matsumoto, M 714

Matsumoto, Y 1349, 1724

Matsumura, Y 593

Matsuura, N 707

Matte, I 989

Matveev, V 1797

Maughan, T 1529

Mauguen, A 1144

Mauland, KK 921

Maurer, D 469

Mauro, DJ 488

Mavroudis, D 316

Maxwell, PH 1151

Maxwell, PJ 480

May, A 1493

May, MT 1755

Mazouni, C 332, 1364

McCall, P 1920

McCarthy, K 1611

McCarthy, NS 338

McClean, M 1896

McConnell, RJ 181

McCormack, L 1602

McCracken, SRC 664

McElligott, AM 281

McFaul, S 620

McGown, G 750

McGuffog, L 1356

McGuinness, DH 1920

McKendick, J 265

McLachlan, J 43

McLaughlin, JR 1906

McLean, C 524

McLennan, J 1384

McMillan, DC 726

Meder, S 138

Medhaoui, D 1517

Medioni, J 1670

Medley, L 1067

Meersma, GJ 1278

Mehta, M 1365

Meijer, A 1278

Meijer, CJLM 685

Meijer, J 227

Meijer, JWR 1020

Mekenkamp, LJM 1020

Melisko, M 1828

Menuel, C 1854

Mesher, D 915

Mesia, R 1691

Metcalfe, K 1384

Meyer, C 1246

Mezei, G 228

Miceli, R 1256

Michael, M 265

Michalski, JM 1365

Michikawa, C 850

Michlmayr, A 543

Middleton, MR 750, 1822

Miida, T 91

Miles, T 1836 
Miller, A 1828

Miller, CJ 971

Miller, CR 545

Mills, R 620

Milne, RL 1356

Milner, AD 265

Minenko, VF 181

Minton, SE 1828

Mints, M 110

Mitchell, E 1697

Mithal, N 620

Miyamoto, Y 1349

Miyata, H 707

Mizota, A 856

Moasser, M 1828

Mohammed, MZ 653

Mole, DJ 1007

Molina-Garrido, MJ 224

Møller, S 419

Möller-Levet, C 971

Molloy, TJ 1913

Montella, M 1207

Moons, KGM 1325

Moore, LE 1797

Moore, N 413

Morel, AN 1067

Moreno, VR 735, 1519

Morgan, C 1755

Morgan, DAL 1393

Morgillo, F 427

Mori, M 707

Morland, R 664

Morrison, DS 726, 1791

Morrison, EE 1602

Moser, R 1529

Moskal, A 1493

Moss, SM 571, 910

Mouw, T 1493

Moyret-Lalle, C 387

Mracek, T 441

Mrkonjic, M 1906

Muir, K 175, 193

Mukherjee, R 1920

Mulder, NH 1193

Mulè, A 1877

Müller-Spahn, C 948

Munneke, BM 488

Munro, AJ 60

Munster, PN 1828

Murata, H 464

Murdoch, J 1836

Muro, K 856

Murphy, MFG 1227

Murray, MJ 746

Murray, PG 1500

Musselman, JRB 214

Nadesan, P 1452

Nagasaka, K 1349

Nagengast, FM 37

Nagtegaal, ID 1020

Nakada, M 798

Nakagawa, H 300

Nakagawa, K 1349, 1594

Nakagawa, M 376, 808

Nakagawa, S 1349

Nakajima, K 707

Nakamura, Y 300
Nakano, R 1882

Nallaswamy, V 1836

Nam, B-H 1126

Nam, J-M 433

Nam, KT 33

Nam, SJ 559

Nam, SY 166

Nambu, E 798

Napolitano, V 427

Narod, SA 1384

Naska, A 1493

Nathan, P 741

Nathanson, KL 1356

Natsugoe, S 714

Nauntofte, B 830

Navarro, C 1493

Navratilova, M 1797

Neal, DE 875, 1656

Negri, E 1207

Negri, FV 1372, 1929

Neoptolemos, JP 1440

Neri, D 1106

Nesland, JM 1434

Nevanlinna, H 1356

Newcomb, PA 763

Newell, DR 1869

Newton, GE 971

Newton, L 1836

$\mathrm{Ng}, \mathrm{CKY} 361$

$\mathrm{Ng}$, LK 460

Ng, SSM 893, 1000

Ngan, S 265

Niault, T 229, 1240

Nichols, PW 1482

Nielsen, FC 830

Nieuwenhuis, MH 37, 1237

Niggemann, B 345

Nishimura, Y 300

Nishio, K 1594

Nishiya, T 593

Nishiyama, K 376, 808

Nistér, M 1747

Nixon, C 664

Nizzoli, R 1372, 1929

Njølstad, TS 921

Noble, TW 1551

Nohata, N 808

Nomura, M 856

Nong, RY 1619

Nonomura, K 241

Norat, T 1493

Nordenskjöld, B 899, 1762

Nørgaard, M 1202

Norrild, B 830

Norvell, J 1628

Novello, S 68

Novotny-Diermayr, V 756

Nuttall, RK 664

Nybo Andersen, A-M 520

Oakley, C 407

Oberg, I 1810

O'Brien, L 175

O'Brien, M 1675

O'Brien, N 620

O'Connor, JPB 719

O'Connor, M 419

Oda, K 1349
Odicino, FE 1418

O’Dwyer, PJ 1862

Oehler, R 543

Offman, J 871

Ogawa, H 505

Ogink, J 227

Ognjanovic, S 532

O'Grady, A 480

Ohga, N 819

Ohyama, Y 850

Okada, N 850

Okamoto, I 1594

Okamoto, W 1594

O'Kane, P 181

Okera, M 407

Oksuzyan, S 228

Olabi, B 1007

Oladipo, O 480

Olesen, F 934, 1249, 1930

Oliphant, R 1791

Oliveira-Cunha, M 514

Oliver, AW 324

Oliver, CT 1356

Oliver, T 620

Olmos, D 1

Olofsson, T 1747

Olopade, OI 1384

Olsen, A 1493

Olsson, A 110

Olver, B 1049

O'Neill, C 1007

Ong, K-r 1356

Orange, C 1920

Orditura, M 427

O'Reilly, DSJ 726

Orlandi, P 1262

Orrego, A 1747

Orsini, N 1196

Osler, M 520

Osorio, A 1356

Österlund, P 599

Osuji, N 407

O’Sullivan, E 1680

Oswal, A 1810

O'Toole, K 1869

O'Toole, L 1529

Otsuka, N 241

Otten, JDM 910

Ottley, CJ 593

Ouafik, L 332

Ouali, M 1544

Oudard, S 1670

Overvad, K 1493

Øyan, AM 921

Paap, E 910

Pagano, JS 1160

Page, E 175

Page, K 1342

Påhlman, L 1619

Pai, SI 1896

Pala, V 1493

Palli, D 1493

Pallone, F 1770

Palumbo, A 1106

Panico, S 1493

Pantel, K 138

Papadaki, C 316
Papadopoulos, P 1675

Papierz, W 968

Paradiso, A 769

Parfrey, PS 1906

Park, JW 166

Park, SR 1126

Park, SY 1730

Park, Y 537

Park, Y-I 1126

Park, YH 559

Parker, T 175

Parmar, M 1529

Parton, M 1675

Pascucci, A 613

Pashayan, N 1656

Passarelli, MN 763

Patel, II 790

Patel, PM 653

Paterson, I 1319

Patil, M 1168, 1822

Paul, AB 1135

Pauwels, P 1472

Pawade, J 1755

Payne, MJ 1822

Paz-Ares, LG 68

Pearmain, P 1505

Pearson, DG 593

Pecorelli, S 1418

Pedersen, AF 1249

Pedersen, L 1202

Peeters, DJE 1472

Peeters, M 1472, 1848

Peeters, PH 1493

Peissel, B 1356

Peleteiro, B 198

Pellegrini, A 248

Penel, N 1544

Peng, G 1649

Peock, S 1356

Pepper, SD 971

Pereira, SP 587

Perks, CM 1587

Persad, RA 1587

Persson, C 1511

Peterlongo, P 1356

Peters, FT 37

Peters, GJ 437, 1185

Petersen, I 1013

Petitpain, N 1238

Peto, J 389

Peto, R 1057

Petrioli, R 613

Pettorelli, E 1079

Pezzella, F 1877

Pflueger, M 469

Pharoah, P 564, 1656

Pharoah, PD 693

Philip, PS 1362

Phillips, K-A 903

Phillips, L 1755

Phillips, M 1246

Piaskowski, S 968

Picard, M 1691

Piché, A 989

Pichler, M 1641

Pickering, L 407

Pigozzo, J 1816

Pinard, M 989 
Pinto, C 427

Pirson, S 948

Pita, G 1356

Plassa, L-F 1739

Plummer, R 593

Pocock, R 175

Pogoda, JM 1482

Pointecouteau, T 155

Pointon, LJ 578

Polesel, J 1207

Pollak, MN 68

Pollett, A 1906

Polterauer, S 223

Polyanskaya, ON 181

Ponchietti, R 613

Pond, GR 1711

Ponnusamy, MP 1038

Pontén, F 1619

Poon, R 1452

Popat, S 407

Porcelli, L 769

Porta, C 1256

Posner, M 1896

Potter, JD 763

Potts, A 1221

Potts, V 1529

Poussa, T 599

Powers, J 756

Powles, T 620, 741

Preiss, L 433

Preston, G 1116

Preston-Martin, S 1482

Pretto, F 1106

Price, N 1459

Price, SJ 1810

Pridgeon, SW 1869

Pring, M 1459

Proby, CM 1459

Procopio, G 1256

Proctor, MJ 726

Pronk, GJ 272

Protheroe, A 620

Protheroe, AS 1822

Prové, A 1472

Provenzano, E 693

Pucciarelli, S 587

Puisieux, A 387

Punt, CJA 1020

Purcell, C 480

Pyko, IV 798

Qi, RZ 841

Qiu, H 208

Qu, M 1747

Quatrale, AE 769

Rachagani, S 1038

Radford, J 719

Radice, P 1356

Raeder, MB 921

Ragnoli, M 1418

Ragoussis, J 1168

Raha, P 1828

Rahman, AA 175

Rahman, S 43

Ramachandran, A 1168

Ramachandran, V 1270

Ramage, JK 587
Ramsay, AK 664

Ramsey-Goldman, R 1478

Ramus, SJ 903

Rancourt, C 989

Rancourt, DE 290

Randolph, SS 1862

Ranson, M 719

Raskett, CM 629

Rasmussen, H 1434

Rau, T 138

Ravaggi, A 1418

Rawson, JB 1906

Raymond, E 272

Razak, ARA 756

Rebbeck, T 1356

Reed, MWR 1551

Reed, N 1067

Reeves, G 1487

Regan, D 886

Reinthaller, A 223

Reisfeld, RA 1410, 1931

Rennert, G 1519

Rennie, IG 1098

Reuschenbach, M 1334

Revaud, D 1056

Rhee, J 1126

Rhee, JC 1027

Riboli, E 1493

Ribom, D 1747

Ricci, S 1256

Rich, AM 460

Richardson, J 1602

Richter, GHS 948

Ridolfi, L 1256, 1816

Ridolfi, R 1816

Riemer, AB 1896

Riemersma, S 1020

Rieske, P 968

Riethdorf, L 138

Riethdorf, S 138

Riis, AH 188, 1804

Rischin, D 265

Ristimäki, A 1890

Rizk, NP 1840

Robertson, C 1221

Robertson, D 188, 1804

Robinson, BA 51

Robinson, M 1319, 1869

Robinson, SP 83

Robinson, V 1007

Robison, LL 532

Röcken, C 1426

Rodgarkia-Dara, C 1303

Rodgers, WH 353

Rodrigues, G 673

Rodríguez, L 1493

Roesler, M 532

Rohrmann, S 1493

Romaguera, D 1493

Romain, S 332

Romani, C 1418

Romanini, A 1816

Romano, N 433

Romanov, GN 181

Ron, E 181

Roos, E 227

Ropert, S 1670

Rosales, R 1434
Rösel, S 1071

Rosell, J 899

Rosen, B 1384

Ross, JA 441, 532

Ross, RK 1482

Rossing, M 830

Rothman, N 1511, 1797

Routledge, P 927

Roviello, F 1770

Rowinsky, EK 488

Rozek, LS 735

Rozendaal, L 685

Rozhko, AV 181

Ruangpratheep, C 1342

Rugo, H 1828

Rumsby, M 673

Ruol, A 427

Russell, I 255

Rydén, M 441

Ryu, HS 1730

Ryu, KW 1126

Ryu, M-H 605

Sablin, M-P 272

Sacco, C 1256

Sadetzki, S 1049

Saeed, S 554

Saida, T 464

Saini, S 892

Sakai, K 1594

Sakaizawa, K 464

Sakata, K-i 1724

Sala, E 361

Salunga, R 1762

Salvatore, L 1262

Salvesen, HB 921

Samonigg, H 1641

Samuel, L 1697

Samuel, S 1270

Sánchez, M-J 1493

Sanders, BG 101

Sandstad, B 1434

Sani, C 248

Sany, O 1505

Sarker, SJ 620

Sasaguri, Y 1882

Sato, H 850

Sawyer, E 175

Scala-Bertola, J 1238

Scarabelli, L 1079

Scartozzi, M 1786

Schaake, E 1325

Schaller, G 1611

Schatzkin, A 537

Schellens, JHM 1913

Schiboni, ML 248

Schirripa, M 1262

Schmitt, J 673

Schoffski, P 1544

Schouten, WR 37

Schuett, W 469

Schulte-Hermann, R 1303

Schüz, J 228

Schwager, K 1106

Schwartz, GK 1862

Sebastian, S 769

Seguí, N 735

Seir, K 1303
Seki, N 376, 808

Selby, P 1529

Sellars, SJ 571

Semba, S 146

Senapati, S 1038

Senju, S 300

Sereno, E 248

Serova, M 272

Severi, G 524

Sexton, JZ 1575

Seymour, GJ 460

Seywright, M 664, 1920

Sezer, O 587

Sgroi, DC 1762

Shah, MA 1840

Shah, S 653

Shahryari, V 308, 892

Shaik, MN 1862

Shak, S 488

Sham, PC 369

Shamash, J 620

Shan, H 505

Sharma, A 1342

Sharp, DM 578

Sharp, L 255

Sharrard, M 673

Shaw, JA 1342

Sheldon, H 1168

Sheng, Q 1241

Sherriff, J 927

Sherrill, B 1848

Shi, Q 1410, 1931

Shibata, N 856

Shiirevnyamba, A 505

Shimada, K 700

Shimajiri, S 1882

Shimizu, C 241

Shin, J-G 605

Shindoh, M 819

Shinohara, N 241

Shirane, A 1349

Shitara, K 856

Shnyder, SD 75

Shoji, K 1349

Shrikant, PA 643

Shu, X-O 1511

Sibbering, DM 1551

Siemerink, EJM 1193

Sieruta, M 968

Siesling, S 1193

Sikand, KA 971

Sileni, VC 427

Sileri, P 1770

Silini, EM 1372, 1929

Sim, MY 941

Sim I, HG 941

Simone, GM 769

Singh, K 308

Sinha, A 1236

Sini, P 769

Sinilnikova, OM 1356

Sinka, K 1221

Sirab, N 1056

Siriwardena, AK 514

Sisley, K 1098

Siu, LL 756

Skeie, G 1493

Skoog, L 1762 
Slamova, A 1797

Slangen, BF 221

Sleijfer, S 1544

Slimani, N 1493

Sloan, P 1319

Sludden, J 593

Smart, L 255

Smith, I 1675

Smith, LD 903

Smith, LM 1038

Smith, MT 635

Smith, RA 1440

Smits, A 1747

Sohail, M 1755

Soliman, H 1739

Soltermann, A 1106

Someya, M 1724

Sommerer, C 1334

Sone, K 1349

Soofi, M 664

Sørensen, HT 188, 1202, 1804

Souglakos, J 316

Souhami, L 1365

Southey, MC 903

Soveri, L-M 599

Spaëth, D 1238

Specht, L 830

Spector, LG 214, 532

Sperati, A 248

Spirtos, NM 353

Spitalieri, G 1686

Spreafico, C 1686

Spurdle, AB 1356

Sriraksa, R 1313

Stål, O 899, 1762

Stanbridge, EJ 353, 841

Stanson, J 1641

Starling, N 1675

Stathopoulos, E 316

Stawski, R 968

Stayner, L-A 756

Stead, M 1529

Stecker, K 1426

Steele, RJC 60

Stefansson, IM 921

Stein, A 886

Stein, GS 629

Stemmler, HJ 1071

Stenmark-Askmalm, M 1356

Stephens, NA 441

Stevens, KN 735

Stevenson, M 480

Steverlynck, C 496

Stewart, D 1529

Stewart, PS 1797

Stewart-Brown, S 175

Stiller, CA 1227

Stockton, DL 60

Stoczynska-Fidelus, E 968

Stone, J 750

Storm, H 178

Stotter, A 1551

Stower, M 673

Stram, DO 1482

Stringfellow, HF 790

Strong, VE 1840

Stuart, N 1535

Stuifbergen, WN 37
Stunnenberg, HG 554

Subjeck, JR 643

Sugimoto, T 376

Suh, SO 892

Suissa, S 1558

Sullivan-Gunn, M 1055

Sun, P 1384

Sundar, S 1505, 1611

Sung, JJY 893

Sung, L 1377

Susumu, N 353

Swanson, J 228

Syed, BM 1393

Sykes, H 407

Szarewski, A 915

Szeszenia-Dabrowska, N 1797

Szybka, M 968

Tabatabai, ZL 308

Tabe, Y 91

Taillibert, S 1854

Takahari, D 856

Takahashi, M 241

Takahashi, T 505

Takata, M 464

Takeda, M 1594

Taketani, Y 1349

Takezawa, K 1594

Takiguchi, S 707

Talamini, R 1207

Talbot, DC 1067, 1822

Talwar, D 726

Tamagnini, I 1372, 1929

Tan, LT 361

Tanaka, Y 892

Tang, J 1440

Tang, LH 1840

Tanikawa, M 1349

Tappenden, N 1680

Targher, G 1234

Tasaki, M 700

Tassi, RA 1418

Tatarano, S 376, 808

Tavani, A 1207

Tavaré, JM 1755

Tavecchio, M 629

Taylor, GA 1869

Taylor, M 1144, 1168, 1822

Taylor, MB 719

Taylor, SE 790

Tebar, M 1020

Teerenstra, S 1020

Tellez, JD 1628

Tempfer, C 223

Temple, J 361

Teng, L 798

Terrazzano, G 1716

Tesch, H 1071

Tesoriero, AA 903

Tessen, HW 1071

Teuffel, O 1377

Teulé, A 1356

Tewari, P 281

Thakker, RV 1067

Thériault, C 989

Therkildsen, MH 830

Thiel, U 948

Thijssen, VL 1185
Thomas, DC 1482

Thomas, EC 1755

Thomas, R 265

Thomas, S 1828

Thomas, W 338

Thomas, ZI 1575

Thompson, D 578

Thomson, S 1810

Thomson, WM 460

Thorncroft, M 750

Thornton, A 255

Thurn, KT 1828

Tian, H 1628

Tilby, MJ 593

Timmerman, D 863

Tinat, J 1517

Tisch, U 1649

Tittarelli, A 228

Tiwary, R 101

Tjønneland, A 1493

To, S-h 369

Todeschini, P 1418

Toffalorio, F 1686

Toki, K 376

Tol, J 1020

Tomas, N 345

Tomita, Y 300

Tomlinson, DC 75

Tomlinson, IP 369

Tørring, ML 934, 1930

Totsuka, Y 819

Tournay, E 1144

Toyota, M 1724

Tran, T 1334

Tranter, N 620

Trayhurn, P 441

Treasure, T 1085

Trevisan, J 790

Trichopoulos, D 1493

Trichopoulou, A 1493

Trigo, JM 1691

Troiani, T 427

Trovik, J 921

Tryfonidis, K 316

Trypaki, M 316

Tsai, H-L 1178

Tsai, MS 982

Tsaroucha, E 316

Tse, LA 208

Tseng, D 1805

Tsujikawa, K 700

Tsukioka, S 1594

Tsunoda, T 300

Tsuruga, T 1349

Tudur-Smith, C 1440

Tumino, R 1493

Tung, N 1384

Turner, EL 875

Turpin, E 1739

Tylko-Hill, K 1836

Uchida, Y 376

Uchikado, Y 714

Uchiyama, A 464

Uehara, H 505

Uen, Y-H 1178

Ueno, K 308

Ueno, S 714
Uenosono, Y 714

Underwood, MA 1920

Ura, T 856

Urbanski, SJ 290

Utsunomiya, S 856

Uzawa, N 850

Uzoh, CC 1587

Valencik, M 1628

Valenti, RM 433

Valentine, HR 971

Valle, L 735, 1519

Vallée, J-N 1854

van Asperen, CJ 1356

van Dalsen, $\mathrm{AD} 37$

van Dam, P-J 1472

van Dam, PA 1472

van Dam, R 37

Van den Eynden, GG 1472

van der Aa, MA 1193

van der Bij, S 1325

van der Bijl, J 37

van der Bilt, ARM 1278

van der Vijgh, WJF 437

van der Zee, AGJ 1278

van Dooren, MF 1356

van Gemert, WG 37

Van Gorp, T 221, 863

van Kemenade, FJ 685

van Krieken, JHJM 1020

Van Laere, SJ 1472

Van Roozendaal, CE 1356

van Schoor, G 910

Vandenberghe, E 281

Vannier, JP 496

van't Veer, LJ 1913

Varna, M 1739

Vasen, HFA 37, 1237

Vasilakis, C 1085

Vasquez-Medrano, DA 1805

Vasse, M 496

Vaughan, V 1055

Vecchione, L 427

Vecht, J 37

Vedsted, P 934, 1249, 1930

Verbeek, ALM 910

Vergote, I 863

Verhoef, S 1356

Vermeulen, PB 1472

Vermorken, JB 1691

Verrando, P 155

Verzoni, E 1256

Vetter, C 345

Vidaud, M 272

Vieth, M 1426

Vijayakrishnan, J 1049

Vimond, N 1144

Vincent, C 635

Vincent, EE 1755

Vinceti, M 228

Vineis, P 1493

Vink-Börger, ME 1020

Vinokurova, S 1334

Visioli, CB 248

Visser, O 685

Vitucci, M 545

Voest, EE 1913

Volkova, E 51 
1940

von Buchwald, C 830

von Deimling, A 1747

von Knebel Doeberitz, M 1334

von Ruesten, A 1493

Voss, MA 1611, 1836

Vreeswijk, M 1356

Vyjayanti, VN 653

Wada-Hiraike, O 1349

Waddell, T 1675

Waisfisz, Q 1356

Walker, AJ 193

Walker, J 915

Walker, JL 353

Walker, L 255, 1356

Walker, LG 578

Walker, RA 1342

Walkington, L 1665

Waller, J 915

Wallgren, A 899

Wallin, A 1196

Wallin, U 1619

Wallis, MG 1680

Walsh, W 756

Walter, K 1296

Wang, J 1848

Wang, J-Y 1178

Wang, K-Y 1882

Wang, L 19

Wang, LD 841

Wang, M 1365

Wang, W 1564

Wang, X-r 208

Wang, X-Y 643

Wang, Y 643

Ward, B 1342

Ward, T 719

Wareham, N 1493

Waring, K 1836

Warmington, S 927

Warwick, J 120

Watanabe, T 146

Watson, AJ 750

Watson, D 488

Watts, C 1810

Waugh, DJJ 480

Waugh, N 255
Webster, J 1665

Weerakkody, RA 1810

Wei, W 1611

Weller, D 1697

Wellman, S 750

Wells, JE 51

Wernli, KJ 763

West, CML 971, 1168, 1319

Wheeler, JL 390

Whiteside, TL 1641

Whitley, GStJ 83

Whynes, D 255

Wieckowski, E 1641

Wiedenmann, B 1426

Wierinckx, A 155

Wiesner, C 469

Wiezorek, J 1848

Wik, E 921

Wilde, DJ 1551

Wilkinson, N 1602

Wilkinson, RA 175

Williams, DC 281

Williams, KP 1575

Williams, L 1007

Williams, RM 746

Willis, JA 51

Wilner, KD 1862

Wilson, C 480

Wilson III, DM 653

Wilson, P 620

Wilson, R 1529

Wilson, RH 480, 593

Wilson, S 927

Winship, I 903

Winterbottom, L 1393

Wirfärt, E 1493

Wishart, GC 564

Wohlberg, M 138

Wolanczyk, M 968

Wolfgang, C 1296

Wolin, KY 882

Wolk, A 1196

Wong, CS 871

Wong, DWM 1393

Wong, EEM 903

Wong, J 1896

Wong, SCC 1000
Wong, VCL 841

Wong, Y-w 369

Wood, J 1459

Wood, NJ 790

Woodman, CBJ 1500

Woolley, C 255

Wu, CW 893

Wu, D-C 1178

Wu, WKK 893

$\mathrm{Wu}, \mathrm{Y} 1882$

Wullschleger, S 1116

Wunsch Filho, V 228

Wyke, S 1697

Xia, L 1270

Xiang, R 1410, 1931

$\mathrm{Xu}, \mathrm{B} 1564$

$\mathrm{Xu}, \mathrm{J} 1401$

Xu, L-A 488

Xu, Y-Z 1869

Yadegarfar, G 587

Yamada, S 1882

Yamaguchi, H 1594

Yamasaki, M 707

Yan, Y 882

Yang, G 1511

Yang, HL 982

Yang, J-H 559

Yang, L 1013

Yang, TYO 4

Yang, W-M 781

Yano, S 505

Yano, T 1349

Yatabe, Y 856

Yau, CC 369

Yau, T-k 369

Ye, W 1511

Yi, J 413

Yip, NC 1564

Yokdang, N 1628

Yokomine, K 300

Yokota, T 856

Yokozaki, H 146

Yoon, J 166

Yoshino, H 376, 808

Yoshizaki, T 1160
Young, JP 1906

Young, L 338

Young, LS 1500

Younghusband, HB 1906

Yousaf, N 1675

Yu, AX 664

Yu, IT-s 208

$\mathrm{Yu}, \mathrm{J} 893$

Yu, W 101

Yuen, C-h 369

Yuen, JSP 941

Yun, J 559

Yung, W-KA 1365

Zablotska, LB 181

Zachariae, R 419, 1249

Zafar, SY 390

Zaffaroni, D 1356

Zahra, MA 361

Zalcberg, JR 265

Zaman, MS 308, 892

Zaniboni, A 1786

Zänker, KS 345

Zanotti, L 1418

Zaridze, D 1797

Zeier, M 1334

Zeller, C 1313

Zhan, Q 1523

Zhang, G 781

Zhang, R-W 781

Zhao, S-G 798

Zhao, Z 1848

Zheng, W 1511

Zheng, Y 763

Zhou, D 24

Zhou, H 1410, 1931

Zhou, W 128

Zhou, Y 91

Zhu, J 756

Zilembo, N 1256

Zironi, S 1079

Zisterer, DM 281

Zöller, K 1013

Zorzi, F 1786

Zucchetto, A 1207

zur Nieden, NI 2901 